DOI: $10.15393 /$ j3.art.2020.6770

UDC 517.968, 517.988

H. K. Kadhim, M. A. Abdul Hussain

\title{
THE ANALYSIS OF BIFURCATION SOLUTIONS OF THE CAMASSA-HOLM EQUATION BY ANGULAR SINGULARITIES
}

\begin{abstract}
This paper studies bifurcation solutions of the CamassaHolm equation by using the local Lyapunov - Schmidt method. The Camassa-Holm equation is studied by reduction to an ODE. We find the key function that corresponds to the functional related to this equation and defined on a new domain. The bifurcation analysis of the key function is investigated by the angular singularities. We find the parametric equation of the bifurcation set (caustic) with its geometric description. Also, the bifurcation spreading of the critical points is found.
\end{abstract}

Key words: Camassa-Holm equation, Bifurcation solutions, Angular Singularities, Caustic

2010 Mathematical Subject Classification: 34K18, $34 K 10$

1. Introduction. The nonlinear problems that occur in mathematics and physics may be formed in the form of operator equations

$$
f(x, \lambda)=b, \quad x \in O, \quad b \in Y, \quad \lambda \in \mathbb{R}^{n},
$$

where $f$ is a smooth Fredholm map with the zero index, $X, Y$ are Banach spaces, and $O \subseteq X$ is open. These problems can be reduced to finitedimensional equations

$$
\Theta(\xi, \lambda)=\beta, \quad \xi \in M, \quad \beta \in N
$$

where $M$ and $N$ are smooth finite-dimensional manifolds. The Lyapunov-Schmidt method can reduce equation (1) to equation (2) so that equation (2) preserves all the analytical and topological features of equation (1) (bifurcation diagram, multiplicity, etc): see, e.g., [8], [12], [10], 
[9]. Singularities of smooth maps play an important role in investigation of bifurcation solutions of boundary-value problems (BVPs). One can find a good review of these studies in [5]. The study of singularities of smooth maps and their applications to the BVPs were significantly developed in the works of Sapronov and his group, for example, in [11], Shvyreva studied the boundary singularities of the function $\widetilde{W}(\eta, \gamma)=\eta_{1}^{4}+\left(c \eta_{1}+\eta_{2}\right)^{2}-2 \varepsilon_{1} \eta_{1}^{2}+2 \varepsilon_{2} \eta_{1}^{2} \eta_{2}+2 \varepsilon_{3} \eta_{1} \eta_{2}+2 \varepsilon_{4} \eta_{1}+2 \varepsilon_{5} \eta_{2}$, where $\eta=\left(\eta_{1}, \eta_{2}\right), \gamma=\left(\varepsilon_{1}, \varepsilon_{2}, \varepsilon_{3}, \varepsilon_{4}, \varepsilon_{5}\right), \eta_{1}, \eta_{2} \geqslant 0$, and considered the functional

$$
V(u, \lambda)=\int_{0}^{\pi}\left(\frac{\left(u^{\prime}\right)^{2}}{2}+\lambda(\cos (u(x))-1)\right) d x,
$$

with the extra condition $\langle u, \omega\rangle=\int_{0}^{\pi} u(x) \omega(x) d x \geqslant 0$, as an application of her results. Abdul Hussain studied in [1] the following problem:

$$
\frac{d^{4} u}{d x^{4}}+\alpha \frac{d^{2} u}{d x^{2}}+\beta u+u^{2}=0, \quad u(0)=u(1)=u^{\prime \prime}(0)=u^{\prime \prime}(1)=0,
$$

with the extra condition $u\left(x_{1}\right) \geqslant 0, u\left(x_{2}\right) \geqslant 0, x_{1}, x_{2} \in[0,1]$, by considering the following functional energy:

$$
V(u, \lambda)=\int_{0}^{1}\left(\frac{\left(u^{\prime \prime}\right)^{2}}{2}-\alpha \frac{\left(u^{\prime}\right)^{2}}{2}+\beta \frac{u^{2}}{2}+\frac{u^{3}}{3}\right) d x,
$$

which is reduced to the study of the following key function with boundaries,

$$
W(\xi, \gamma)=\frac{\xi_{1}^{3}}{3}+\xi_{1} \xi_{2}^{2}+\delta \xi_{2}^{2}+\beta \xi_{1}+o\left(|\xi|^{3}\right)+O\left(|\xi|^{3}\right) O(\gamma),
$$

where $\xi_{1}-a \xi_{2} \geqslant 0, \xi_{1}+b \xi_{2} \geqslant 0$. In this paper, we consider a boundaryvalue problem not previously studied in the same way; see Section 3.Details on the purpose of the paper are also given there.

The Lyapunov-Schmidt method supposes that $f: \Omega \subset E \rightarrow F$ is a smooth nonlinear Fredholm map of zero index. The map $f$ is said to have the variational property, when there exists a functional $V: \Omega \subset E \rightarrow \mathbb{R}$, such that $f=\operatorname{grad}_{H} V$ or, equivalently, $\frac{\partial V}{\partial x}(x, \lambda) h=\langle f(x, \lambda), h\rangle_{H}, \forall x \in \Omega$, 
$h \in E$, where $\langle\cdot, \cdot\rangle_{H}$ is the scalar inner product in the Hilbert space $H$. Also, it assumes that $E \subset F \subset H$. The solutions of equation $f(x, \lambda)=0$ are the critical points of functional $V(x, \lambda)$. The finite-dimensional reduction method (the Lyapunov-Schmidt method) can reduce the problem $V(x, \lambda) \rightarrow \operatorname{extr}, x \in E, \lambda \in \mathbb{R}^{n}$ to the equivalent problem $W(\xi, \lambda) \rightarrow$ extr, $\xi \in \mathbb{R}^{n}$, where $W(\xi, \lambda)$ is called the key function. If $N=\operatorname{span}\left\{e_{1}, \ldots, e_{n}\right\}$ is a subspace of a Banach space $E$, where $\left\{e_{1}, \ldots, e_{n}\right\}$ is an orthonormal set in $H$, then the key function $W(\xi, \lambda)$ can be defined by the form $W(\xi, \lambda)=\inf _{x:\left\langle x, e_{i}\right\rangle=\xi_{i}} V(x, \lambda), \xi=\left(\xi_{1}, \ldots, \xi_{n}\right)$. The function $W$ preserves all topological and analytical properties of the functional $V$ (multiplicity, bifurcation diagram, etc) [9]. The study of bifurcating solutions of the functional $V$ is tantamount to the study of bifurcating solutions of the key function. If $f$ possesses a variational property, then the equation $\Theta(\xi, \lambda)=\operatorname{grad} W(\xi, \lambda)=0$ is called the bifurcating equation.

Definition 1. [4] The set of all $\lambda$ for which the function $f(x, \lambda)$ possesses degenerate critical points is called the bifurcation set (caustic) and denoted by $\Sigma$.

2. Angular singularities of Fredholm functionals [4]. To investigate the behavior of a Fredholm functional in a neighborhood of an angular singular point, one uses the reduction to an analogous extremal problem

$$
W(x) \rightarrow \text { extr }
$$

where $x \in C, C=\left\{x=\left(x_{1}, x_{2}, \ldots, x_{m}, x_{m+1}, \ldots, x_{m+t}, \ldots, x_{n}\right)^{\top} \in \mathbb{R}^{n}\right.$ : $\exists$ integers $t, m \ni 0 \leqslant t \leqslant n-m$ and $\left.x_{m} \geqslant 0, x_{m+1} \geqslant 0, \ldots, x_{m+t} \geqslant 0\right\}$. We say that a point $a \in C$ is conditionally critical for a smooth function $W$ in $\mathbb{R}^{n}$ if grad $W(a)$ is orthogonal to the least face of $C$ containing $a$, and the set $\left\{\left(x_{m}, x_{m+1}, \ldots, x_{m+t}\right)^{\top} \in \mathbb{R}^{n}: x_{m} \geqslant 0, x_{m+1} \geqslant 0, \ldots, x_{m+t} \geqslant 0\right\}$ is called the $m$-hedral angle.

The multiplicity of the conditionally critical point $a$ (denoted by $\bar{\mu}$ ) is the dimension of the quotient algebra: the set

$$
\widehat{Q}=\frac{\prod_{a}\left(\mathbb{R}^{n}\right)}{I}
$$

where $\prod_{a}\left(\mathbb{R}^{n}\right)$ is the ring of germs of smooth functions on $\mathbb{R}^{n}$ at the point 
$a$ and

$$
I:= \begin{cases}\left(\frac{\partial W}{\partial x_{1}}, \ldots, x_{m} \frac{\partial W}{\partial x_{m}}, \ldots, x_{m+t} \frac{\partial W}{\partial x_{m+t}}, \ldots, \frac{\partial W}{\partial x_{n}}\right), & \text { for all } m>1, \\ \left(x_{m} \frac{\partial W}{\partial x_{m}}, \ldots, x_{m+t} \frac{\partial W}{\partial x_{m+t}}, \ldots, \frac{\partial W}{\partial x_{n}}\right), & \text { for } m=1\end{cases}
$$

is the angular Jacobi ideal in $\prod_{a}\left(\mathbb{R}^{n}\right)$. The multiplicity $\bar{\mu}$ of a conditionally critical point $a$ is equal to the sum of multiplicities $\mu+\mu_{0}$, where $\mu$ is the (usual) multiplicity of $W$ on $\mathbb{R}^{n}$, while $\mu_{0}$ is the (usual) multiplicity of the restriction $W \mid \partial C$ (where $\partial C$ is the boundary of the set $C$ ).

If a critical point is "usual", then spreadings of bifurcating extremes (bif-spreadings) are represented by the row $\left(c_{0}, c_{1}, \ldots, c_{n}\right)$, where $c_{i}$ is the number of critical points of the Morse index $i$. If we are dealing with an angular (or boundary) critical point, then bif-spreadings are represented by the following matrix of order $(m+t+1) \times(n+1)$ :

$$
\left(\begin{array}{ccccc}
c_{0}^{1} & c_{1}^{1} & \cdot & \cdot & c_{n}^{1} \\
c_{0}^{2} & c_{1}^{2} & \cdot & \cdot & c_{n}^{2} \\
\cdot & \cdot & \cdot & \cdot & \cdot \\
\cdot & \cdot & \cdot & \cdot & \cdot \\
c_{0}^{m+t} & c_{1}^{m+t} & \cdot & \cdot & c_{n}^{m+t} \\
c_{0} & c_{1} & \cdot & \cdot & c_{n}
\end{array}\right) .
$$

Here $c_{i}^{j}$ is the number of the angular critical points of index $i$ (for $j=1,2, \ldots, m+t$ ), while $c_{i}$ is the number of usual (situated inside $C$ ) critical points of index $i$.

\section{Bifurcation solutions of the Camassa-Holm equation.}

In 1993, Camassa and Holm used the Hamiltonian method to derive a new formula for a completely integrable shallow water wave equation

$$
u_{t}+2 k u_{x}-u_{x x t}+3 u u_{x}=2 u_{x} u_{x x}+u u_{x x x}
$$

where $u$ is the fluid speed in the $x$ direction (or, equivalently, the height of the waters free surface above a flat bottom), $k$ is a constant related to the critical depthless water wave velocity, and subscripts indicate partial derivatives. This equation keeps the higher-order terms (the right-hand side of (3)) in a little-amplitude expansion of the incompressible Euler conditions for unidirectional motion of wave at the free surface under the 
effect of gravity. Now, equation (3) is called the equation of CamassaHolm $(\mathrm{CH})$. In recently years, $\mathrm{CH}$ equation has been generalized to the general Camassa-Holm (GCH) equation:

$$
u_{t}+2 k u_{x}-u_{x x t}+\frac{1}{2}[f(u)]_{x}=2 u_{x} u_{x x}+u u_{x x x}
$$

where $f(u)$ is a function of $u$ and $[f(u)]_{x}$ is the derivative of $f$ with respect to $x$. In [6], [7], the authors investigated bifurcations of the traveling-wave solutions for the general Camassa-Holm equation (3) and the corresponding traveling-wave system with $f(u)=\alpha u^{2}+\beta u^{3}$. Note that we can obtain equation (3) from equation (4) by assuming $\alpha=3$ and $\beta=0$ in the function $f(u)=\alpha u^{2}+\beta u^{3}$.

Suppose that $u(x, t)=z(y), y=x-\alpha t$, where $\alpha$ is the wave speed. Equation (3) can be converted to an ODE with the variable $z(y)$ :

$$
\alpha z^{\prime \prime}+\beta z+\frac{3}{2} z^{2}-\left(\frac{1}{2}\left(z^{\prime}\right)^{2}+z z^{\prime \prime}\right)=0
$$

where $\alpha, \beta$ are parameters and $\iota=d / d y$.

In this section, we investigate bifurcation solutions of equation (5) with the boundary conditions that satisfy equation (5):

$$
z(0)=z(1)=0
$$

where $z=z(y), y \in[0,1]$.

Assume that $f: E \rightarrow M$ is a nonlinear Fredholm operator with zero index, from the Banach space $E$ to the Banach space $M, E=C^{2}([0,1], \mathbb{R})$ is the space of all continuous functions that have derivatives of order at least two, $M=C^{0}([0,1], \mathbb{R})$ is the space of all continuous functions, and $f$ is defined by the operator equation:

$$
f(z, \lambda)=\alpha z^{\prime \prime}+\beta z+\frac{3}{2} z^{2}-\left(\frac{1}{2}\left(z^{\prime}\right)^{2}+z z^{\prime \prime}\right)=0,
$$

where $\lambda=(\alpha, \beta)$. Every solution of the equation (5) (1-periodic solution) is a solution of the operator equation (6). Since the operator $f$ possesses the variational property, there exists a functional $V$, such that

$$
f(z, \lambda)=\operatorname{grad}_{H} V(z, \lambda),
$$


where

$$
V(z, \lambda)=\frac{1}{2} \int_{0}^{1}\left(-\alpha\left(z^{\prime}\right)^{2}+\beta z^{2}+z^{3}+z\left(z^{\prime}\right)^{2}\right) d x,
$$

$\operatorname{grad}_{H} V$ denotes the gradient of $V$, and $z$ fulfills the following conditions:

$$
\begin{aligned}
& \left\langle e_{1}, z\right\rangle+a\left\langle e_{2}, z\right\rangle \geqslant 0, \\
& \left\langle e_{1}, z\right\rangle-a\left\langle e_{2}, z\right\rangle \geqslant 0
\end{aligned}
$$

Here $\left\{e_{1}, e_{2}\right\} \subset E$ is an orthonormal set in the Hilbert space $H,\langle\cdot, \cdot\rangle$ is the scalar inner product in $H$, and $a$ is a positive real constant.

In this case, every solution of equation (6) is a critical point of the functional $V$.

The purpose of this paper is to find the solution areas of equation (5), where each bifurcating solution of (5) equals a critical point of the functional $V$ and each critical point of the functional $V$ coincides with a critical point of the key function of the functional $V$. Therefore, in subsections below we investigate bifurcating extremes of the function (8), equivalent to investigating bifurcating extremes of functional $V$ by the key function (i.e., studying bifurcating solutions of functional $V$ is tantamount to studying bifurcating solutions of this function). Hence, studying bifurcating solutions of equation (5) is equivalent to studying bifurcating solutions of function (8).

3.1 Singularities of the function of codimension three. In this subsection, we consider the function that has codimension three at the origin [2]. It is defined by

$$
W(s, \rho)=\frac{\eta_{1}^{3}}{3}+\frac{\eta_{2}^{3}}{3}+\eta_{2} \eta_{1}^{2}+\eta_{2}^{2} \eta_{1}+\lambda_{1} \eta_{2} \eta_{1}+\lambda_{2}\left(\eta_{1}^{2}+\eta_{2}^{2}\right)
$$

where $s=\left(\eta_{1}, \eta_{2}\right), \eta_{1}, \eta_{2} \geqslant 0, \rho=\left(\lambda_{1}, \lambda_{2}\right)$ and $\lambda_{1}, \lambda_{2}$ are parameters.

The function (8) has codimension three at the origin, hence it has multiplicity four. The main purpose is to find a geometrical description (a bifurcation diagram) of the caustic of function (8), and then to determine the spreading of the critical points of this function. The germ (the principle part) of function (8) is

$$
W_{0}=\frac{\eta_{1}^{3}}{3}+\frac{\eta_{2}^{3}}{3}
$$


So, from section we have $I=\left(\eta_{1} \frac{\partial W_{0}}{\partial \eta_{1}}, \eta_{2} \frac{\partial W_{0}}{\partial \eta_{2}}\right)=\left(\eta_{1}^{3}, \eta_{2}^{3}\right)$, and $\bar{\mu}=9$ where $\mu=3$ and $\mu_{0}=6$. Since multiplicity $\bar{\mu}$ is equal to the number of critical points [3], the number of critical points of function (8) is nine; three of these points lie on the boundary $\eta_{1}=0$, three points lie on the boundary $\eta_{2}=0$, and three points lie in the interior. So, the caustic of function (8) is the union of six sets:

$$
\Sigma=\Sigma_{0,0} \cup \Sigma_{0,1}^{i n t} \cup \Sigma_{0,1}^{e x t} \cup \Sigma_{1,0}^{i n t} \cup \Sigma_{1,0}^{e x t} \cup \Sigma_{1,1},
$$

where $\Sigma_{0,0}$ is the subset (component) of the caustic corresponding to the degeneration at the vertex $(0,0) ; \Sigma_{0,1}^{i n t}$ and $\Sigma_{0,1}^{e x t}$ are the subsets (components) of the caustic corresponding to the degeneration of boundary singularities along the boundary $\eta_{1}=0$ and along the normal, respectively; $\Sigma_{1,0}^{i n t}$ and $\Sigma_{1,0}^{e x t}$ are the same for the boundary $\eta_{2}=0$ and its normal; finally, $\Sigma_{1,1}$ is the component corresponding to the degeneration of the interior (non-boundary) critical points.

3.2. Degeneration at the vertex $(0,0)$. To determine the set $\Sigma_{0,0}$, we must find the following union:

$$
\left\{\left(\lambda_{1}, \lambda_{2}\right): \frac{\partial W\left(0,0, \lambda_{1}, \lambda_{2}\right)}{\partial \eta_{1}}=0\right\} \bigcup\left\{\left(\lambda_{1}, \lambda_{2}\right): \frac{\partial W\left(0,0, \lambda_{1}, \lambda_{2}\right)}{\partial \eta_{2}}=0\right\}
$$

Since the conditions in the two sets above are satisfied for all pairs $\left(\lambda_{1}, \lambda_{2}\right)$ in the plane, there is no graphical representation of the set $\Sigma_{0,0}$, so it is empty.

3.3. Degeneration along the boundary $\eta_{1}=0$ (internal degeneration). The next lemma gives the equation that describes the set $\Sigma_{0,1}^{\text {int }}$.

Lemma 1. The parametric equation that represents the set $\Sigma_{0,1}^{i n t}$ is

$$
\lambda_{2}^{2}=0
$$

Proof. To determine the set $\Sigma_{0,1}^{\text {int }}$, we consider the boundary critical points of function (8), such that the second-order partial derivative of this function with respect to $\eta_{2}$ vanishes at these points, i.e, the following relations are valid:

$$
\frac{\partial W\left(0, \eta_{2}, \lambda_{1}, \lambda_{2}\right)}{\partial \eta_{2}}=\frac{\partial^{2} W\left(0, \eta_{2}, \lambda_{1}, \lambda_{2}\right)}{\partial \eta_{2}^{2}}=0, \quad \eta_{2}>0
$$


or

$$
\eta_{2}^{2}+2 \eta_{2} \lambda_{2}=\eta_{2}+\lambda_{2}=0
$$

We can represent the relations above as the system of equations

$$
\begin{aligned}
\eta_{2}^{2}+2 \eta_{2} \lambda_{2} & =0 \\
\eta_{2}+\lambda_{2} & =0
\end{aligned}
$$

From equation (9b), we get $\eta_{2}=-\lambda_{2}$, and substituting the value of $\eta_{2}$ in equation (9a), we find

$$
\lambda_{2}^{2}=0
$$

which represents the set $\Sigma_{0,1}^{i n t}$.

3.4. Degeneration along the boundary $\eta_{1}=0$ (external degeneration). The next lemma gives the equation that describes the set $\Sigma_{0,1}^{e x t}$.

Lemma 2. The parametric equation that represents the set $\Sigma_{0,1}^{e x t}$ is

$$
\left(-2 \lambda_{2}+\lambda_{1}\right) \lambda_{1}=0
$$

Proof. To determine the set $\Sigma_{0,1}^{e x t}$, we consider the boundary critical points of function (8), such that the first-order partial derivative of this function with respect to $\eta_{1}$ vanishes at these points, i. e, the following relations are valid:

$$
\frac{\partial W\left(0, \eta_{2}, \lambda_{1}, \lambda_{2}\right)}{\partial \eta_{2}}=\frac{\partial W\left(0, \eta_{2}, \lambda_{1}, \lambda_{2}\right)}{\partial \eta_{1}}=0, \quad \eta_{2}>0
$$

or

$$
\eta_{2}^{2}+2 \eta_{2} \lambda_{2}=\eta_{2}^{2}+\eta_{2} \lambda_{1}=0
$$

We can represent the above relations by the system of equations

$$
\begin{aligned}
\eta_{2}^{2}+2 \eta_{2} \lambda_{2} & =0 \\
\eta_{2}^{2}+\eta_{2} \lambda_{1} & =0
\end{aligned}
$$

Since $\eta_{2}>0$, by the above assumption, equation (10b) yields only $\eta_{2}=-\lambda_{1}$; substituting the value of $\eta_{2}$ in equation (10a), we get the equation

$$
\left(-2 \lambda_{2}+\lambda_{1}\right) \lambda_{1}=0 .
$$


3.5. Degeneration along the boundary $\eta_{2}=0$ (internal degeneration). To determine the set $\Sigma_{1,0}^{i n t}$, we consider the boundary critical points of function (8), such that the second-order partial derivative of this function with respect to $\eta_{1}$ vanishes at these points, i. e, the following relations are valid:

$$
\frac{\partial W\left(\eta_{1}, 0, \lambda_{1}, \lambda_{2}\right)}{\partial \eta_{1}}=\frac{\partial^{2} W\left(\eta_{1}, 0, \lambda_{1}, \lambda_{2}\right)}{\partial \eta_{1}^{2}}=0, \quad \eta_{1}>0
$$

or

$$
\eta_{1}^{2}+2 \eta_{1} \lambda_{2}=2 \eta_{1}+2 \lambda_{2}
$$

Lemma 3. The parametric equation cwfor the set $\Sigma_{1,0}^{i n t}$ is

$$
\lambda_{2}^{2}=0
$$

Proof. The proof is similar to the proof of Lemma 1.

3.6. Degeneration along the boundary $\eta_{2}=0$ (external degeneration). To determine the set $\Sigma_{1,0}^{e x t} \mathrm{r}$, consider the boundary critical points of function (8), such that the first-order partial derivative of this function with respect to $\eta_{2}$ vanishes at these points, i.e, the following relations are valid:

$$
\frac{\partial W\left(\eta_{1}, 0, \lambda_{1}, \lambda_{2}\right)}{\partial \eta_{1}}=\frac{\partial W\left(\eta_{1}, 0, \lambda_{1}, \lambda_{2}\right)}{\partial \eta_{2}}=0, \eta_{1}>0
$$

or

$$
\eta_{1}^{2}+2 \eta_{1} \lambda_{2}=\eta_{1}^{2}+\eta_{1} \lambda_{1}=0
$$

Lemma 4. The parametric equation that represents the set $\Sigma_{1,0}^{e x t}$ is

$$
\left(-2 \lambda_{2}+\lambda_{1}\right) \lambda_{1}=0
$$

Proof. The proof is similar to the proof of Lemma 2.

3.7. Degeneration of the interior (non-boundary). The next lemma gives the equation that represents the set $\Sigma_{1,1}$.

Lemma 5. The parametric equation that represents the set $\Sigma_{1,1}$ is

$$
\lambda_{1}^{3}+2 \lambda_{1}^{2} \lambda_{2}-4 \lambda_{1} \lambda_{2}^{2}-8 \lambda_{2}^{3}=0 .
$$


Proof. To determine the set $\Sigma_{1,1}$, consider the critical points of function (8), defined by the system

$$
\frac{\partial W\left(\eta_{1}, \eta_{2}, \lambda_{1}, \lambda_{2}\right)}{\partial \eta_{1}}=\frac{\partial W\left(\eta_{1}, \eta_{2}, \lambda_{1}, \lambda_{2}\right)}{\partial \eta_{2}}=0, \eta_{1}, \eta_{2}>0 .
$$

or

$$
\eta_{1}^{2}+2 \eta_{1} \eta_{2}+2 \eta_{1} \lambda_{2}+\eta_{2}^{2}+\eta_{2} \lambda_{1}=\eta_{1}^{2}+2 \eta_{1} \eta_{2}+\eta_{1} \lambda_{1}+\eta_{2}^{2}+2 \eta_{2} \lambda_{2}=0
$$

Then, make the Hessian determinant of function (8) vanish to get the equation

$$
-4 \eta_{1} \lambda_{1}+8 \eta_{1} \lambda_{2}-4 \eta_{2} \lambda_{1}+8 \eta_{2} \lambda_{2}-\lambda_{1}^{2}+4 \lambda_{2}^{2}=0
$$

We can express (11) and (12) in the following system:

$$
\begin{aligned}
\eta_{1}^{2}+2 \eta_{1} \eta_{2}+2 \eta_{1} \lambda_{2}+\eta_{2}^{2}+\eta_{2} \lambda_{1} & =0 \\
\eta_{1}^{2}+2 \eta_{1} \eta_{2}+\eta_{1} \lambda_{1}+\eta_{2}^{2}+2 \eta_{2} \lambda_{2} & =0 \\
-4 \eta_{1} \lambda_{1}+8 \eta_{1} \lambda_{2}-4 \eta_{2} \lambda_{1}+8 \eta_{2} \lambda_{2}-\lambda_{1}^{2}+4 \lambda_{2}^{2} & =0
\end{aligned}
$$

By subtracting equation (13b) from equation (13a), we get

$$
\left(-\lambda_{1}+2 \lambda_{2}\right) \eta_{1}+\left(\lambda_{1}-2 \lambda_{2}\right) \eta_{2}=0
$$

This implies $\left(\eta_{1}-\eta_{2}\right)\left(-\lambda_{1}+2 \lambda_{2}\right)=0$ and, in turn, gives the system

$$
\begin{aligned}
\eta_{1} & =\eta_{2}, \\
\left(-\lambda_{1}+2 \lambda_{2}\right) & =0,
\end{aligned}
$$

Solving (14a) together with (13c), we obtain $\eta_{1}=\eta_{2}=-\frac{\left(\lambda_{1}+2 \lambda_{2}\right)}{8}$. Substituting the values of $\eta_{1}$ and $\eta_{2}$ to equation (13a), we get

$$
\lambda_{1}^{2}+4 \lambda_{2} \lambda_{1}+4 \lambda_{2}^{2}=0 .
$$

Multiplying (14b) by (15) yields $\lambda_{1}^{3}+2 \lambda_{1}^{2} \lambda_{2}-4 \lambda_{1} \lambda_{2}^{2}-8 \lambda_{2}^{3}=0$.

Theorem 1. Parametric equation of the bifurcation set (caustic) of function (8) is given by the equation

$$
\left(\lambda_{1}^{3}+2 \lambda_{1}^{2} \lambda_{2}-4 \lambda_{1} \lambda_{2}^{2}-8 \lambda_{2}^{3}\right)\left(-2 \lambda_{2}+\lambda_{1}\right)^{2} \lambda_{1}^{2} \lambda_{2}^{4}=0
$$


Proof. Since, the caustic of function (8) consists of the union of six sets,

$$
\Sigma=\Sigma_{0,0} \cup \Sigma_{0,1}^{i n t} \cup \Sigma_{0,1}^{e x t} \cup \Sigma_{1,0}^{i n t} \cup \Sigma_{1,0}^{e x t} \cup \Sigma_{1,1},
$$

so, the parametric equation of the caustic is composed of the product of all left-hand sides of the equations for the caustic components, which is equal to zero. Since the equations of the caustic components were found in lemmas (1), (2), (3), (4), and (5), the equation

$$
\left(\lambda_{1}^{3}+2 \lambda_{1}^{2} \lambda_{2}-4 \lambda_{1} \lambda_{2}^{2}-8 \lambda_{2}^{3}\right)\left(-2 \lambda_{2}+\lambda_{1}\right)^{2} \lambda_{1}^{2} \lambda_{2}^{4}=0 .
$$

represents the parametric equation of the bifurcating set (caustic) of function (8).

Proposition 1. If the condition $\lambda_{2}<\frac{-1}{2} \lambda_{1}, 0 \neq \lambda_{2} \neq \frac{1}{2} \lambda_{1}$, is satisfied, function (8) has three non-degenerate real critical points (one interior and two boundary points: one point on $\eta_{1}=0$ and the other on $\eta_{2}=0$ ).

Proof. The critical points of function (8) can be expressed as follows:

$$
\begin{array}{llrl}
P_{1} & =\left(\frac{-1}{4} \lambda_{1}-\frac{1}{2} \lambda_{2}, \frac{-1}{4} \lambda_{1}-\frac{1}{2} \lambda_{2}\right), & P_{2} & =\left(0,-2 \lambda_{2}\right), \\
P_{3} & =\left(-2 \lambda_{2}, 0\right), & P_{4}=(0,0) .
\end{array}
$$

The point $P_{1}$ is a non-degenerate real point, in the interior of the domain of function (8), if the conditions $\frac{-1}{4} \lambda_{1}-\frac{1}{2} \lambda_{2}>0$ and $\left(\lambda_{1}-2 \lambda_{2}\right)$ $\left(\lambda_{1}+2 \lambda_{2}\right) \neq 0$ are satisfied. These conditions imply $\lambda_{2}<\frac{-1}{2} \lambda_{1}$ and $\lambda_{2} \neq \frac{1}{2} \lambda_{1}$, otherwise $P_{1}$ can nott be a non-degenerate real point in the interior of the domain of function (8). Clearly, the two points $P_{2}$ and $P_{3}$ are non-degenerate boundary real points for all $0 \neq \lambda_{2} \neq \frac{1}{2} \lambda_{1}$ (the point $P_{2}$ lies on $\eta_{1}=0$ and the point $P_{3}$ lies on $\left.\eta_{2}=0\right)$. The point $P_{4}$ is a vertical point (i.e., it is neither an interior point nor a boundary point). This completes the proof.

Theorem 2. The matrices of bif-spreadings of the critical points of function (8) are as follows:

$$
\left(\begin{array}{lll}
0 & 0 & 0 \\
0 & 0 & 0 \\
1 & 0 & 0
\end{array}\right),\left(\begin{array}{lll}
1 & 0 & 0 \\
1 & 0 & 0 \\
0 & 0 & 0
\end{array}\right),\left(\begin{array}{lll}
1 & 0 & 0 \\
1 & 0 & 0 \\
0 & 1 & 0
\end{array}\right),\left(\begin{array}{lll}
0 & 1 & 0 \\
0 & 1 & 0 \\
1 & 0 & 0
\end{array}\right) .
$$

Proof. The caustic equation, which we have found in Theorem (1), can be represented geometrically: see Figure 1. This figure decomposes the plane 


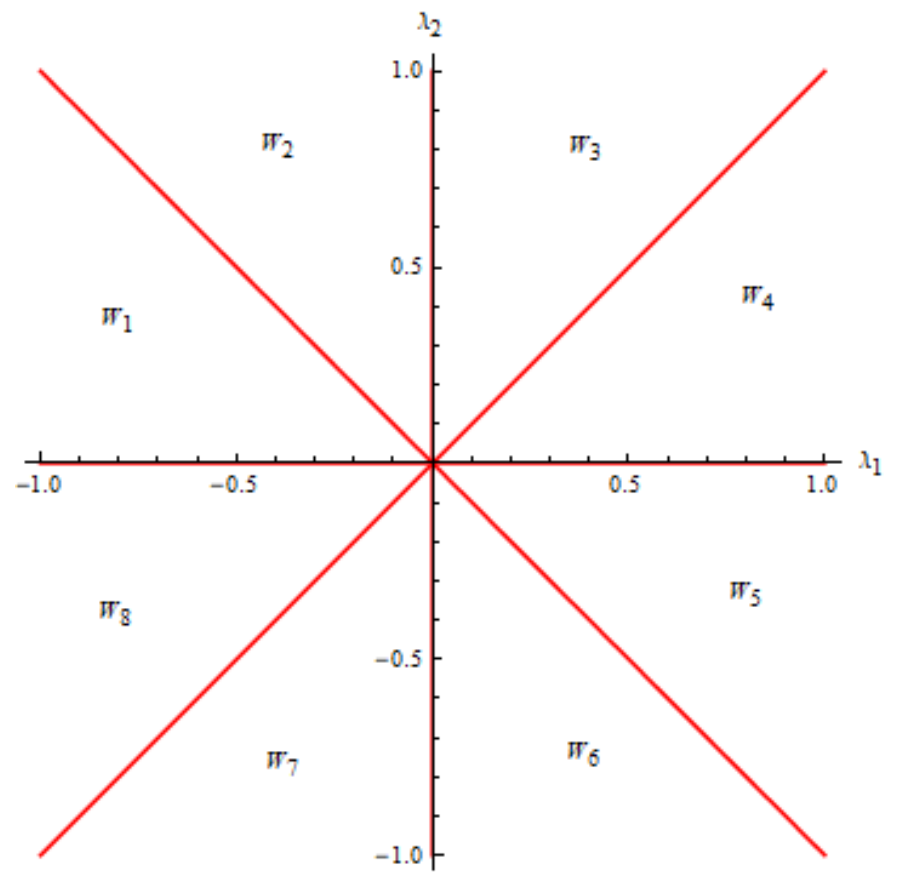

Figure 1: Describes the caustic of function (8) in $\lambda_{1} \lambda_{2}$-plane

of parameters into eight regions $W_{i}, i=1,2, \ldots, 7,8$; every region contains a fixed number of non-degenerate real critical points. These points are divided into internal and boundary points. The quality of the internal points can be determined using the second-derivative test, while the quality of the boundary points can be determined using the Morse lemma [4]. Hence,the spreading of the critical points is as follows: if the parameters $\lambda_{1}, \lambda_{2}$ belong to

1) $W_{1}$ : then we have one interior minimum critical point;

2) $W_{5}$ : then we have two critical points (one minimum point on the boundary $\eta_{1}=0$ and one minimum point on the boundary $\eta_{2}=0$ );

3) $W_{6}$ or $W_{7}$ : then we have three critical points (one minimum point on the boundary $\eta_{1}=0$, one minimum point on the boundary $\eta_{2}=0$, and one interior saddle point);

4) $W_{8}$ : then we have three critical points (one saddle point on the boundary $\eta_{1}=0$, one saddle point on the boundary $\eta_{2}=0$, and one interior minimum point); 
5) $W_{2}$ or $W_{3}$ or $W_{4}$ : then we have no real critical points in the domain $\left\{\left(\eta_{1}, \eta_{2}\right): \eta_{1} \geqslant 0, \eta_{2} \geqslant 0\right\}$.

Using this, we get the matrices of bif-spreadings as is described in (16).

In addition, the values of the Morse index at a given vertex that corresponds to one of the previous eight regions are defined as follows: index $=0 \Leftrightarrow \lambda_{1}, \lambda_{2}$ belong to any region of the previous eight regions.

In figure 2, parts (a), (b), (c), and (d) show the locations of contour lines with respect to the boundaries of the domain of function (8), number and type of critical points corresponding for all regions in the caustic of function (8); (a) corresponds to $W_{1}$, (b) corresponds to $W_{5}$, (c) corresponds to $W_{6}$ or $W_{7}$, and (d) corresponds to $W_{8}$.

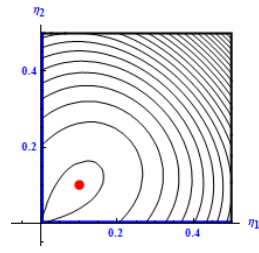

(a)

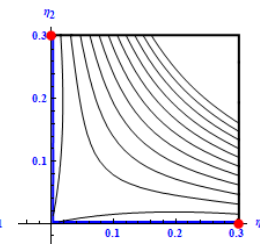

(b)

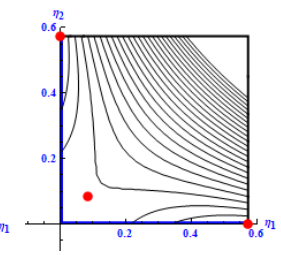

(c)

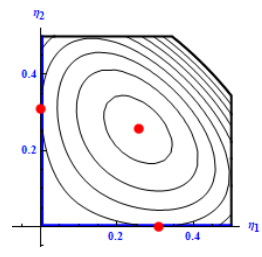

(d)

Figure 2

Therefore, in subsections below we investigate bifurcating extremes of the function (8), equivalent to investigating bifurcating extremes of functional $V$ by the key function In the following theorem, we prove that investigating of bifurcating extremes of functional $V$ is reduced to investigating of bifurcating extremes of function (8).

Theorem 3. The normal form of the key function $W_{1}$ corresponding to the functional $V$ is given by

$$
W_{1}(y, \rho)=\frac{\eta_{1}^{3}}{3}+\frac{\eta_{2}^{3}}{3}+\eta_{2} \eta_{1}^{2}+\eta_{2}^{2} \eta_{1}+\lambda_{1} \eta_{2} \eta_{1}+\lambda_{2}\left(\eta_{1}^{2}+\eta_{2}^{2}\right)
$$

where $y=\left(\eta_{1}, \eta_{2}\right), \eta_{1}, \eta_{2} \geqslant 0, \rho=\left(\lambda_{1}, \lambda_{2}\right)$ and $\lambda_{1}, \lambda_{2}$ are the parameters. Proof. By using the Lyapunov-Schmidt scheme, the linearized equation corresponding to the equation $(6)$ at the point $(0, \lambda)$ gets the form

$$
\begin{aligned}
& A h=0, \quad h \in E, \\
& h(0)=h(1)=0,
\end{aligned}
$$


where $A=\alpha \frac{d^{2}}{d x^{2}}+\beta$.

The solution of the linearized equation that satisfies also the initial conditions is given by $e_{p}(x)=c_{p} \sin (p \pi x), p=1,2, \ldots$, and the characteristic equation corresponding to this solution is

$$
-\alpha(p \pi)^{2}+\beta=0
$$

This equation defines characteristic lines $\ell_{p}$ in the $\alpha \beta$-plane. The characteristic lines $\ell_{p}$ consist of the points $(\alpha, \beta)$, for which the linearized equation has non-zero solutions [10]. The point of intersection of the characteristic lines in the $\alpha \beta$-plane is the bifurcation point, so the bifurcation point for the equation $(6)$ is $(\alpha, \beta)=(0,0)$. The following values of the parameters $\alpha, \beta: \alpha=0+\delta_{1}, \beta=0+\delta_{2}$, where $\delta_{1}$ and $\delta_{2}$ are small parameters, lead to a bifurcation along the modes $e_{1}(x)=c_{1} \sin (\pi x)$, $e_{2}(x)=c_{2} \sin (2 \pi x)$. Since $\left\|e_{1}\right\|=\left\|e_{2}\right\|=1$, we have $c_{1}=c_{2}=\sqrt{2}$.

Let $N=\operatorname{Ker}(A)=\operatorname{span}\left\{e_{1}, e_{2}\right\}$; then the space $E$ can be decomposed in the direct sum of two subspaces, $N$ and the orthogonal complement to $N$ :

$$
E=N \oplus N^{\perp}, N^{\perp}=\left\{v \in E: \int_{0}^{1} v e_{k} d x=0, k=1,2\right\} .
$$

Similarly, the space $M$ can be decomposed in the direct sum of two subspaces, $N$ and the orthogonal complement to $N$ :

$$
M=N \oplus \tilde{N}^{\perp}, \tilde{N}^{\perp}=\left\{\omega \in M: \int_{0}^{1} \omega e_{k} d x=0, k=1,2\right\} .
$$

There exist two projections $P: E \rightarrow N$ and $I-P: E \rightarrow N^{\perp}$, such that $P z=\omega$ and $(I-P) z=v(I$ is the identity operator $)$. Hence, every vector $z \in E$ can be written in the form

$$
z=\omega+v
$$

where $\omega=x_{1} e_{1}+x_{2} e_{2} \in N, v \in N^{\perp}, x_{i}=\left\langle z, e_{i}\right\rangle$. Thus, by the implicit function theorem, there exists a smooth map $\Theta: N \rightarrow N^{\perp}$, such that

$$
\widetilde{W}(w, \gamma)=V(\Theta(w, \gamma), \gamma)
$$




$$
w=\left(x_{1}, x_{2}\right), \gamma=\left(\delta_{1}, \delta_{2}\right) .
$$

Then the key function $\widetilde{W}$ can be written in the the form

$$
\begin{aligned}
\widetilde{W}(w, \gamma)=V\left(x_{1} e_{1}+x_{2} e_{2}+\Theta\right. & \left.\left(x_{1} e_{1}+x_{2} e_{2}, \gamma\right), \gamma\right)= \\
& =W_{2}(w, \gamma)+o\left(|w|^{3}\right)+O\left(|w|^{3}\right) O(\gamma)
\end{aligned}
$$

where,

$$
\begin{aligned}
W_{2}(w, \gamma)=\left(\frac{4}{3} \frac{\sqrt{2}}{\pi}+\frac{2}{3} \sqrt{2} \pi\right) & x_{1}^{3}+\left(\frac{16 \sqrt{2}}{5 \pi}+\frac{24 \sqrt{2} \pi}{5}\right) x_{2}^{2} x_{1}+ \\
+ & \left(\frac{-1}{2} \alpha \pi^{2}+\frac{\beta}{2}\right) x_{1}^{2}+\left(-2 \alpha \pi^{2}+\frac{\beta}{2}\right) x_{2}^{2}
\end{aligned}
$$

Now, substitute the value of $z$ in (17) in the conditions (7a) and (7b), respectively, to get

$$
\begin{aligned}
& x_{1}+a x_{2} \geqslant 0, \\
& x_{1}-a x_{2} \geqslant 0 .
\end{aligned}
$$

Changing variables, we get $\eta_{1}=\frac{x_{1}+a x_{2}}{2}, \eta_{2}=\frac{x_{1}-a, x_{2}}{2}$. Solving for $x_{1}$ and $x_{2}$ yields the following: $x_{1}=\eta_{1}+\eta_{2}$, and $x_{2}=\frac{\eta_{1}-\eta_{2}}{a}$; substituting them in the function $W_{2}$, we have

$$
\begin{aligned}
W_{2}(q, \gamma)= & \left(\frac{4}{3} \frac{\sqrt{2}}{\pi}+\frac{2}{3} \sqrt{2} \pi+\frac{\frac{16}{5} \frac{\sqrt{2}}{\pi}+\frac{24}{5} \sqrt{2} \pi}{a^{2}}\right) \eta_{1}^{3}+ \\
& +\left(\frac{4}{3} \frac{\sqrt{2}}{\pi}+\frac{2}{3} \sqrt{2} \pi+\frac{\frac{16}{5} \frac{\sqrt{2}}{\pi}+\frac{24}{5} \sqrt{2} \pi}{a^{2}}\right) \eta_{2}{ }^{3}+ \\
& +\left(\frac{4 \sqrt{2}}{\pi}+2 \sqrt{2} \pi-\frac{\frac{16}{5} \frac{\sqrt{2}}{\pi}+\frac{24}{5} \sqrt{2} \pi}{a^{2}}\right) \eta_{2} \eta_{1}^{2}+ \\
& +\left(\frac{4 \sqrt{2}}{\pi}+2 \sqrt{2} \pi-\frac{\frac{16}{5} \frac{\sqrt{2}}{\pi}+\frac{24}{5} \sqrt{2} \pi}{a^{2}}\right) \eta_{2}{ }^{2} \eta_{1}+ \\
& +\left(-\alpha \pi^{2}+\beta-\frac{2\left(-2 \alpha \pi^{2}+\frac{1}{2} \beta\right)}{a^{2}}\right) \eta_{2} \eta_{1}+ \\
+\left(-\frac{1}{2} \alpha \pi^{2}+\right. & \left.\frac{1}{2} \beta+\frac{-2 \alpha \pi^{2}+\frac{1}{2} \beta}{a^{2}}\right) \eta_{1}{ }^{2}+\left(-\frac{1}{2} \alpha \pi^{2}+\frac{1}{2} \beta+\frac{-2 \alpha \pi^{2}+\frac{1}{2} \beta}{a^{2}}\right) \eta_{2}{ }^{2}
\end{aligned}
$$

where $q=\left(\eta_{1}, \eta_{2}\right)$ and $\eta_{1}, \eta_{2} \geqslant 0$. 
The geometrical form of bifurcations of critical points and the first asymptotics of branches of bifurcating for the function $\widetilde{W}$ are completely determined by its principal part $W_{2}$. If we replace $\eta_{1}$ and $\eta_{2}$ by

$$
\frac{\sqrt[3]{5}}{\sqrt{2}} \sqrt[3]{\frac{\pi a^{2}}{5 \pi^{2} a^{2}+36 \pi^{2}+10 a^{2}+24}} \eta_{1} \text { and } \frac{\sqrt[3]{5}}{\sqrt{2}} \sqrt[3]{\frac{\pi a^{2}}{5 \pi^{2} a^{2}+36 \pi^{2}+10 a^{2}+24}} \eta_{2}
$$

in the function $W_{2}$, respectively, then $W_{1}$ and $W_{2}$ are contact equivalence, since in this case they have the same germ (the same principal part)

$$
W_{0}\left(\eta_{1}, \eta_{2}\right)=\frac{\eta_{1}^{3}}{3}+\frac{\eta_{2}^{3}}{3}
$$

and the same deformation. Therefore, the caustic of the function $W_{2}$ coincides with the caustic of the function $W_{1}$.

Thus, the function $W_{1}$ has all the topological and analytical properties of the functional $V$, so the bifurcation analysis of equation (6) is equivalent to the study of bifurcation analysis of the function $W_{1}$. This shows that the study of bifurcations of extremes of the functional $V$ is reduced to the study of bifurcation of extremes of function (8).

Acknowledgment. The authors would like to express their hearty thanks to the referees for their valuable suggestions and comments for revising the manuscript.

\section{References}

[1] Abdul Hussain M. A. Corner Singularities of Smooth Functions in the Analysis of Bifurcations Balance of the Elastic Beams and Periodic Waves. $\mathrm{PhD}$ thesis, Voronezh Univ., Voronezh, 2005 (in Russian).

[2] Arnold V. I, Gusein-Zade S. M., Varchenko A. N. Singularities of Differential Maps. Springer, 1988.

DOI: https://doi.org/10.1007/978-1-4612-3940-6.

[3] Berczi G. Lectures on Singularities of Maps. Trinity Term. Oxford, 2010.

[4] Darinskii B. M., Tcarev C. L., Sapronov Yu. I. Bifurcations of Extremals of Fredholm Functionals. J. Math. Sci., 2007, vol. 145, pp. 5311-5453.

DOI: https://doi.org/10.1007/s10958-007-0356-2.

[5] Golubitsky M. , Schaeffer D. G. Singularities and Groups in Bifurcation Theory. vol. I (Applied Mathematical Sciences 51), Springer, 1985.

DOI: https://doi.org/10.1007/978-1-4612-5034-0. 
[6] Li J., Qiao Z. Bifurcation and Exact Traveling Wave Solutions for a Generalized Camassa-Holm Equation. Internat. J. Bifur. Chaos Appl. Sci. Engrg., 2013, vol. 23, no. 03, Article ID: 1350057.

DOI: https://doi.org/10.1142/S0218127413500570.

[7] Li J. Singular Nonlinear Travelling Wave Equations: Bifurcations and Exact Solutions. Science Press, Beijing, 2013.

[8] Loginov B. V. Theory of Branching Nonlinear Equations in The conditions of Invariance Group. Tashkent. Fan, 1985.

[9] Sapronov Yu. I. Regular Perturbation of Fredholm Maps and Theorem about odd Field. Tr. Mat. Fak. Voronezh. Gos. Univ., 1973, vol. 10, pp. 82-88.

[10] Sapronov Yu. I. Finite Dimensional Reduction in the Smooth Extremely Problems. Uspekhi Mat. Nauk, 1996, vol. 51, issue 1(307), pp. 101-132. DOI: http://dx.doi.org/10.1070/RM1996v051n01ABEH002741.

[11] Shveriova O. V. Caustic and bif-spreadings for a boundary extremal with a triple degeneration along the boundary. Tr. Mat. Fak. Voronezh. Gos. Univ., 2002, no. 7, pp. 149-160 (in Russian).

[12] Vainberg M. M., Trenogin V. A. Theory of Branching Solutions of Nonlinear Equations. Bull. Amer. Math., Soc., 1975, vol. 81, pp. 886-889.

DOI: https://doi.org/10.1090/S0002-9904-1975-13871-7.

Received July 22, 2019.

In revised form, January 21, 2020.

Accepted January 26, 2020.

Published online February 16, 2020.

Faculty of Education for Pure Sciences

Department of Mathematics

University of Basrah

Basrah, Iraq.

E-mail:

Hussein K. Kadhim

khashanhussein@gmail.com

Mudhir A. Abdul Hussain

mud_abd@yahoo.com 